\title{
MIGRACIÓN INTERNA Y MOVILIDAD OCUPACIONAL EN LA CIUDAD DE MÉXICO*
}

\author{
Humberto MUÑoz \\ Universidad Nacional Autónoma de México \\ Orlandina de Oliveira \\ El Colegio de México
}

\section{INTRODUCCIÓN}

EN ESTE ARTículo se analizan algunas tendencias de la movilidad ocupacional experimentada por varias cohortes de migrantes y nativos en la Ciudad de México. ${ }^{1}$

Un análisis descriptivo de este tema es un principio importante en el conocimiento de las relaciones del fenómeno migratorio con la estructura ocupacional y la movilidad. A través del estudio de varias cohortes, es posible conocer las diferencias sucesivas de la población en el momento en que entra al mercado de trabajo y, a partir de ahí, sus cambios ocu. pacionales. ${ }^{2} \mathrm{El}$ análisis intrageneracional de dichos cambios permite ilus-

* Este trabajo fue presentado en su versión inicial a la III Reunión del Grupo de Trabajo sobre Migraciones Internas de la Comisión de Población y Desarrollo, cLAcso, celebrada en Santiago de Chile del 11 al 15 de diciembre de 1972. Los autores desean agradecer a los miembros del grupo las sugerencias que hicieron durante la discusión, las cuales fueron incorporadas en gran parte en el texto. Asimismo, agradecen a Harley Browning, Waltraut Feindt y Claudio Stern sus valiosos comentarios.

1 Este artículo se basa en los datos de la primera fase del proyecto de investigación sobre migración interna, estructura ocupacional y movilidad social en el área metropolitana de la Ciudad de México realizado conjuntamente por el Instituto de Investigaciones Sociales de la Universidad Nacional Autónoma de México y el Centro de Estudios Económicos y Demográficos de El Colegio de México.

Los datos se obtuvieron de una muestra representativa del área metropolitana de la Ciudad de México, la cual consistió de 2500 viviendas. La muestra se diseñó con base en una estratificación habitacional en función del tipo y las características de la vivienda, los servicios urbanos, etc. En forma aleatoria, y de acuerdo al peso relativo de cada estrato en la población total, se seleccionaron 500 manzanas en las cuales se hizo un listado de viviendas de donde se eligieron cinco en cada una de ellas. La cédula de entrevista fue diseñada para recoger información sobre todas las personas que vivían habitualmente en "cada vivienda. La encuesta se realizó entre noviembre de 1969 y febrero de 1970.

2 Un análisis de movimientos entre grupos ocupacionales específicos se encuentra en el libro de J. Balán, H. Browning y E. Jelín, Men in a Developing Society: Geographic and Social Mobility in Monterrey, Mexico, University of Texas "Press, 1973, caps. V y VIII. En relación con el problema puede verse E. Jelín, "Estructura ocupacional, cohortes y ciclo vital". Actas de la Primera Conferencia Regional Latinoamericana de Población, México, El Colegio de México, 1971, vol. II, pp. 97-102. 
trar en forma bastante clara las relaciones de la migración y la movilidad, pues es razonable suponer que las diferencias en la incorporación a la fuerza de trabajo y la movilidad de migrantes y nativos varían de acuerdo con las características de cada cohorte.

El estudio de la movilidad se efectúa tomando como base una escala ocupacional que sirve para registrar los movimientos verticales. Para establecer la jerarquía ocupacional se utilizaron criterios que reflejan características de los individuos en la estructura socio-económica, tales como la propiedad o el uso de bienes en el trabajo, el número de personas que se emplean o que se tienen bajo responsabilidad y algunos otros. ${ }^{3}$

Se advierte que al resumir toda la información en un conjunto de estratos, se limitan las posibilidades de captar otros movimientos que son importantes para comprender el proceso de formación y los cambios de la mano de obra. De este modo, los movimientos intra e intersectoriales de la fuerza de trabajo deberán abordarse posteriormente.

\section{MigRación, ESTRUCTURA OCUPACIONAL Y MOVILIDAD}

En algunos estudios sobre América Latina, ${ }^{4}$ se afirma que el desarrollo económico ha contribuido al cambio de las estructuras ocupacionales en las ciudades a través del incremento de actividades industriales y la expansión de los servicios ligados a éstas, tales como los de tipo financiero, bancario, los de comercialización, administración, etc. Ello implicó un aumento de las proporciones de fuerza de trabajo que se dedican a las actividades manuales industriales y a ocupaciones no manuales, como profesionistas, técnicos, personal directivo, etc. Se sostiene también que dichas tendencias fueron acompañadas por el crecimiento de actividades del sector terciario menos productivas como son, entre otras, las de servicios personales y las de comercio ambulante.

Como afirma Singer, ${ }^{5}$ el empleo relativo en el sector terciario se expande en virtud de dos procesos que en el fondo son contradictorios. Por un lado, el desarrollo genera un aumento de la demanda de servicios de producción (comercio, transportes y comunicaciones) y de servicios de consumo colectivo (gobierno y actividades sociales) y, por el otro, la presión de la oferta de la fuerza de trabajo genera los servicios de consumo individual, en los cuales gran parte de las personas ocupadas se encuentran en el servicio doméstico remunerado.

Cuando se analiza la composición ocupacional de la población económicamente activa (PEA) masculina de 21 a 60 años de edad en la

3 En el apéndice metodológico se explica con mayor amplitud la forma como fue construida la escala ocupacional.

1 Véase Fernando $H$. Cardoso $v$ José Luis Reyna, "Industrialización, estructura ocupacional y estratificación social en América Latina" en el libro del primer autor, Cuestiones de sociología del desarrollo, Santiago de Chile, Editorial Universitaria, 1968, pp. 68-105; Paul Singer, "Migraciones internas: Consideraciones teóricas sobre su estudio", en Migración y Desarrollo, CLACSO, 1972 y; Projecto de pesquisa do sector terciario da área metropolitana de Sáno Paulo (Mimeo), 1972.

5 Véase Paul Singer, Força de trabalho e emprego no Brasil, 1920-1969. Cuderno Núm. 3. CEBRAP, São Paulo, Brasil, 1971, p. 13. 
Ciudad de México, se encuentra que la estructura ocupacional presenta características que reflejan las tendencias mencionadas.

En el cuadro 1 puede observarse que las ocupaciones no manuales tales como profesionistas, técnicos y agentes de ventas, constituyen un 44.1\% de la población considerada. Esto refleja la ampliación de las oportunidades de empleo en estas categorías, lo que está relacionado con los movimientos de mano de obra resultantes de cambios estructurales.

\section{Cuadro 1}

Área Metropolitana de la Ciudad de México: Distribución de la POBLACIÓN MASCULINA ECONÓMICAMENTE ACTIVA DE 21 A 60 AÑOS DE EDAD, POR CONDICIÓN MIGRATORIA Y GRUPOS OCUPACIONALES, 1970

(Porcientos)

\begin{tabular}{|c|c|c|c|}
\hline $\begin{array}{c}\text { Grupos } \\
\text { ooupropionales }\end{array}$ & Xigrantese & Mativas & Total \\
\hline 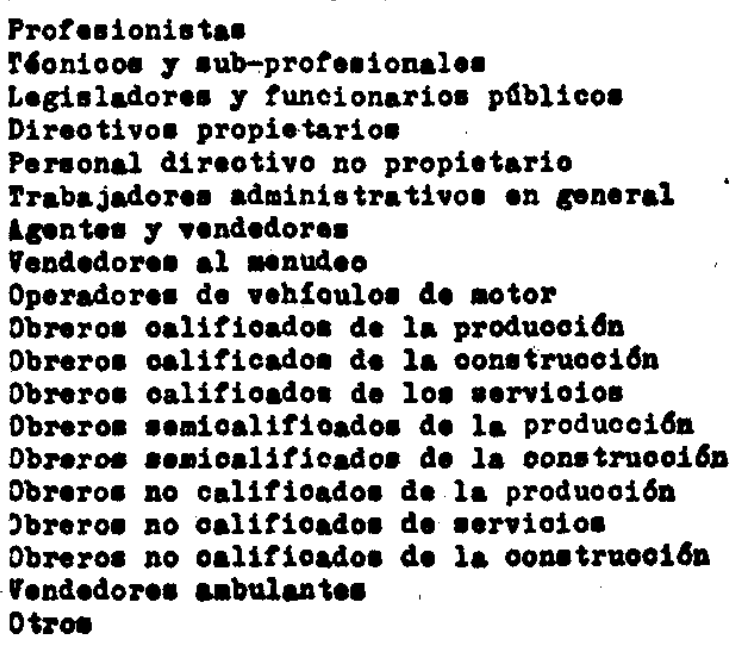 & $\begin{array}{r}5.2 \\
6.4 \\
0.6 \\
8.6 \\
6.6 \\
6.6 \\
2.8 \\
4.4 \\
5.9 \\
7.1 \\
0.6 \\
5.7 \\
12.7 \\
3.0 \\
9.2 \\
8.0 \\
2.2 \\
2.6 \\
2.0\end{array}$ & $\begin{array}{r}3.7 \\
7.9 \\
0.2 \\
9.8 \\
4.9 \\
11.5 \\
3.6 \\
5.0 \\
6.7 \\
11.1 \\
0.7 \\
3.7 \\
12.6 \\
1.4 \\
7.4 \\
5.7 \\
1.0 \\
1.4 \\
2.0\end{array}$ & $\begin{array}{r}4.4 \\
7.2 \\
0.4 \\
9.1 \\
5.6 \\
9.4 \\
3.3 \\
4.7 \\
6.3 \\
9.4 \\
0.6 \\
4.6 \\
12.6 \\
2.1 \\
8.2 \\
6.7 \\
1.5 \\
1.9 \\
2.0\end{array}$ \\
\hline Yotal & 100.2 & 100.3 & 100.0 \\
\hline
\end{tabular}

Asimismo, el hecho de que el grupo de obreros de la producción sea más numeroso, ya que alcanza un $30.2 \%$ del total, da idea del impacto que ha tenido el crecimiento industrial en la configuración de la estructura ocupacional.

Los obreros de servicios y de la construcción representan $15.5 \%$ de la población analizada, de los cuales más de la mitad (el $8.2 \%$ ) son no calificados. Los trabajadores no calificados de los servicios $(6.7 \%)$ comprenden a los limpiabotas, ayudantes de cantineros, mozos, etc., y los de la construcción $(1.5 \%)$ a los que trabajan como peones. Si a éstos se les agregan los vendedores ambulantes $(1.9 \%)$ y los obreros no calificados de la producción $(8.2 \%)$ se obtiene un panorama de lo que puede significar la marginalidad ocupacional en la ciudad. Casi una quinta parte de la PEA masculina de 21 a 60 años de edad se ubica en posiciones marginales, es decir, en ocupaciones cuyos niveles de ingresos son muy bajos y con escasas o nulas prestaciones. ${ }^{6}$

6 Estos grupos ocupacionales fueron definidos como "marginales" en un trabajo anterior de los autores con Claudio Stern, "Migración y marginalidad ocupacional en la Ciudad de México", El perfil de México en 1980, III, México, Siglo XXI Editores, 1972. 
En resumen, podría señalarse que los cambios que produce el desarrollo económico sobre la estructura ocupacional permiten, por un lado, el surgimiento de sectores de clase media y, por el otro, contribuyen a la formación de ocupaciones marginales. Estos cambios en la estructura ocupacional pueden ser mejor comprendidos cuando se analizan en relación con las migraciones internas, como mecanismos de desplazamiento geográfico de la fuerza de trabajo, y con la movilidad estructural de grupos sociales en los centros urbanos.

Así, por ejemplo, la migración interna, principalmente la rural-urbana, puede ser entendida como un mecanismo de transferencia de mano de obra no calificada a las grandes ciudades. En estos términos, determinados flujos migratorios, que aumenten la oferta de mano de obra, pueden llevar al incremento o persistencia de actividádes económicas poco productivas, como los servicios personales, comercio ambulante y otras. ${ }^{7}$

En otro trabajo sobre ${ }^{8}$ la Ciudad de México, se afirma que las corrientes migratorias coadyuvan a que la oferta de mano de obra sobrepase la capacidad del sistema económico para generar empleos, lo cual ayuda a explicar la creciente marginalización de ciertos sectores de la PEA.

Si se observa la distribución de la población migrante ${ }^{9}$ y nativa a lo largo de la estructura ocupacional (véase el cuadro 1), puede apreciarse que la primera es algo más numerosa en las ocupaciones marginales, aunque también entre los profesionistas y el personal directivo no propietario, que son quienes constituyen los grupos de más altos ingresos de toda la población económicamente activa (superior a $\$ 500$ mensuales).$^{10}$ Este resultado reafirma la idea de que la población migrante es heterogénea y sugiere que sólo una parte de la oferta de mano de obra, resultante de los flujos migratorios, ha contribuido a la ampliación de actividades marginales, ya que por otra parte es posible que se incorpore a ocupaciones no manuales o a manuales calificadas.

Sin embargo, la distribución actual de la población migrante y nativa en la estructura ocupacional, resulta del nivel ocupacional al que se incorporó a la fuerza de trabajo y de la movilidad ocupacional experimentada desde entonces.

7 Véase J. R. Brandão Lopes, Desenvolvimento e mudança social. São Paulo; Companhia Editora Nacional, 1971; Anibal Quijano, Redefinición de la dependencia y proceso de marginalización en América Latina, Santiago, ILPES (Mimeo), 1970 y; Paul Singer, "Migraciones Internas...", op. cit., 1972.

8 Véase H. Muñoz, O. de Oliveira y C. Stern, op. cit., 1972.

9 Los migrantes fueron clasificados según su comunidad de origen, esto es, aquella en la que el individuo pasó la mayor parte del tiempo entre los 5 y los 15 años de edad. Se consideró como migrante a toda persona cuya comunidad de origen no es el área metropolitana de la Ciudad de México. La categoría de nativo incluye a toda persona cuya comunidad de origen es el mencionado centro urbano. Para mayores detalles técnicos sobre este aspecto consúltese H. Muñoz, O. de Oliveira y C. Stern, "Categorías de migrantes y nativos y algunas de sus características socio-económicas: Comparaciones entre las Ciudades de Monterrey y México". Revista Paraguaya de Sociología, año 8, Núm. 21, 1971. Para una discusión acerca de la importancia de esta definición, véase $\mathrm{H}$. Browning y W. Feindt, "Diferencias entre la población nativa y la migrante en Monterrey", Demografía y Economía, El Colegio de México, Vol. II, Núm. 2, 1968, pp. 183-204.

10 En el trabajo de los autores y C. Stern, "Migración..." loc. cit., 1972, se presenta en el apéndice núm. 2 un cuadro resumen con información spbre el pro. medio de ingreso y educación de todos los grupos ocupacionales. 
Así por ejemplo, es posible suponer que una parte de la mano de obra proveniente de la migración, haya pasado por medio de desplazamientos ocupacionales o sectoriales, a niveles superiores a los que se incorporó inicialmente, lo cual ha permitido que los migrantes se encuentren distribuidos a todo lo largo de la estructura ocupacional urbana.

\section{Tendencias diferenciales DE LA MOVIlidad ENTRE LA POBla- CIÓN MIGRANTE Y NATIVA 11}

La descripción de estos aspectos tiene por objeto conocer algunas tendencias diferenciales de ascenso entre la población migrante y nativa en el área metropolitana de la Ciudad de México.

La movilidad se analiza de forma intrageneracional, comparando el nivel de la primera ocupación con el nivel de la ocupación actual de la población entrevistada. ${ }^{12}$

Con este fin se han jerarquizado las ocupaciones de acuerdo a una escala de seis niveles. Las ocupaciones no manuales como profesionistas, técnicos, funcionarios públicos, directivos propietarios, trabajadores administrativos, vendedores, etc., se agruparon en ocupaciones no manuales de nivel alto, medio y bajo según diversos criterios, como por ejemplo, el hecho de ser o no propietario, el número de personas remuneradas que se emplean, o el número de personas supeditadas directa o indirectamente, etc. Las ocupaciones manuales como operadores de vehículos de motor, obreros de la construcción y de la producción fueron agrupadas en manuales calificados, semicalificados y no calificados. En el caso de los trabajadores manuales, además de los criterios ya mencionados, se establecieron otros a partir del título de las ocupaciones, el grado de responsabilidad por el uso o manejo de maquinaria y las exigencias de capacitación para realizar las tareas. ${ }^{13}$

Cuando se compara la ocupación de los migrantes, cuyo primer trabajo fue en la Ciudad de México, con la primera ocupación de los nati-

11 El tema de la migración y la movilidad ocupacional ha sido poco estudiado en México. El análisis más completo es el que se hizo en Monterrey cuyos resultados se presentan en el trabajo de J. Balán, H. Browning y E. Jelín, op. cit. Este análisis es de suma importancia ya que los autores contaban con una historia de vida de los entrevistados, lo cual les permitió establecer en qué momento del ciclo vital ocurre la migración a la ciudad y definir diferentes momentos en la vida de los sujetos para estudiar la movilidad. En otros términos, se analizó en forma dinámica la relación entre migración, edad al migrar y movilidad ocupacional.

12 La primera ocupación ha sido definida como aquella que tuvo el entrevistad̆o por primera vez durante cuatro meses seguidos, o más, en un año, contados a partir del momento en que empezó a trabajar. Esto elimina, en parte, el riesgo de tomar en cuenta ocupaciones que se ejercieron de manera eventual durante períodos muy cortos de tiempo, las cuales en ocasiones pertenecen a bajos estratos, lo que contribuye a elevar los montos de movilidad. Asimismo, es necesario señalar que la metodología para el análisis de la movilidad es sumamente compleja y que el hecho de que entre la población exista mucha o poca movilidad depende, en cierta forma, de los métodos que se utilicen para la medición. Como en este trabajo se comparan la primera ocupación con la actual, las posibilidades de movilidad para la población tienden a ser máximas.

13 Para mayores detalles técnicos sobre la construcción de los estratos y sobre la distribución de la PEA masculina de 21 a 60 años, por estratos, véase el apéndice metodológico. 
vos (véase el cuadro 2) se encuentra que los migrantes iniciaron su vida activa en la ciudad dentro de los estratos no manuales en proporciones ligeramente superiores que los últimos. El $23.8 \%$ de migrantes que no habían trabajado antes de venir a la Ciudad de México comenzaron a hacerlo en el estrato de los no manuales bajos, en contra de un $19.1 \%$ de los nativos. Asimismo, un $4.9 \%$ de migrantes comenzó a trabajar en el estrato de los no manuales altos por un $1.6 \%$ de nativos.

En contraste con lo anterior, cuando se observa la distribución por estrato de la primera ocupación de la población migrante que empezó a trabajar fuera de la Ciudad de México (véase el cuadro 2), se aprecia que la mayor proporción $(68.1 \%)$ se ubicaba entre los manuales no calificados, y que esta proporción es bastante mayor que la de los nativos que comenzaron a trabajar en ocupaciones incluidas en dicho estrato $(48.6 \%)$.

\section{Cuadro 2}

Área Metropolitana de la Ciudad de México: Distribución de la PEA MASCULINA DE 21 A 60 AÑOS DE EDAD, SEGÚN CONDICIÓN MIGRATORIA, LUGAR Y ESTRATO DE LA PRIMERA OCUPACIÓN

(Porcientos)

\begin{tabular}{|c|c|c|c|c|c|}
\hline \multirow{2}{*}{$\begin{array}{l}\text { Eatrato primere } \\
\text { ooupeo16n }\end{array}$} & \multicolumn{3}{|c|}{ Migrantegal } & \multirow[b]{2}{*}{ Hativos } & \multirow[b]{2}{*}{ Total } \\
\hline & $\begin{array}{l}\text { 18. ooupaoibn } \\
\text { on Ia } \\
\text { Cd. de Moxico }\end{array}$ & $\begin{array}{l}\text { ogupao16n } \\
\text { fuera de le } \\
\text { cd. de KCxioo }\end{array}$ & $\begin{array}{l}\text { Sub- } \\
\text { total }\end{array}$ & & \\
\hline $\begin{array}{l}\text { Do manual } \\
\text { Alto } \\
\text { Modio } \\
\text { Bejo }\end{array}$ & $\begin{array}{r}\frac{4.9}{2.2} \\
23.8 \\
\end{array}$ & $\begin{array}{l}1.4 \\
1.5 \\
8.7\end{array}$ & $\begin{array}{r}2.4 \\
1.8 \\
12.9\end{array}$ & $\begin{array}{r}\frac{1.6}{1.7} \\
19.1 \\
\end{array}$ & $\begin{array}{r}1.9 \\
1.7 \\
16.4\end{array}$ \\
\hline $\begin{array}{l}\text { Manual } \\
\text { Gallficado } \\
\text { senioalifiondo } \\
\text { Ho califioado }\end{array}$ & $\begin{array}{l}12.8 \\
13.6 \\
42.7\end{array}$ & $\begin{array}{l}10.0 \\
10.2 \\
68.1 \\
\end{array}$ & $\begin{array}{l}10.6 \\
10.9 \\
61.4\end{array}$ & $\begin{array}{l}16.5 \\
12.9 \\
48.6\end{array}$ & $\begin{array}{l}13.7 \\
12.1 \\
54.1\end{array}$ \\
\hline Total & 100.0 & 100.0 & 100.0 & 100.4 & 99.9 \\
\hline
\end{tabular}

a Representan $43.3 \%$ de la PEA masculina de 2 T a 60 años de edad.

b Representan $72.5 \%$ de la PEA masculina migrante de 21 a 60 años de edad.

Así, la población migrante tiene una composición diferencial muy marcada en lo que se refiere a su primera ocupación, lo que refleja la diversidad de grupos sociales que han participado en los movimientos migratorios hacia la Ciudad de México. Las características de dichos flujos migratorios tales como el tipo y tamaño de la localidad de nacimiento, el nivel de educación, las experiencias ocupacionales previas y la época de llegada, ${ }^{14}$ junto con los cambios socioeconómicos ocurridos

14 Un 58\% de los migrantes de la PEA masculina de 21 a 60 años de edad, de la Ciudad de México, nacieron en comunidades rurales (menos de 5000 habitantes), $19 \%$ en comunidades urbanas pequeñas (de 5000 a menos de 20000 habitantes), $19 \%$ en urbanas medianas (de 20000 a menos de 100000 habitantes) y $4 \%$ en urbanas grandes (100000 o más habitantes). Véase el trabajo de los autores con C. Stern, "Categorías de..." loc cit., 1971. Cálculos más recientes indican que un $52.1 \%$ de los migrantes de la PEA masculina de 21 a 60 años de edad llegaron por última vez a la Ciudad de México entre los 15 y 24 años. Asimismo, $\mathbf{7 2 . 5} \%$ tuvo su primera ocupación fuera de la Ciudad de México y $32.9 \%$ comenz6 su vida activa en ocupaciones agrícolas. 
en la Ciudad de México en los últimos decenios, pueden haber influido en las diferencias de movilidad ocupacional de la población migrante y entre ésta y la de los nativos. ${ }^{15}$

A continuación se analizan dichas diferencias atendiendo exclusivamente a los movimientos ascendentes, ya que la baja proporción de personas que ha experimentado una movilidad descendente y la similitud de las proporciones de ésta entre las categorías migratorias, no ameritan mayor análisis por el momento (entre los migrantes un $7.5 \%$ son móviles descendentes y entre los nativos un 8.6\%).

La movilidad se estudia a partir de cada uno de los estratos de incorporación, con el objeto de conocer los ascensos de migrantes y nativos que comenzaron a trabajar en un mismo nivel ocupacional. ${ }^{16}$

Al controlar el estrato de entrada a la vida activa (véase el cuadro 3) los porcientos de migrantes y nativos con movilidad ascendente presentan dos tendencias básicas:

a) Los migrantes que comenzaron a trabajar en ocupaciones manuales no calificadas o semicalificadas tienden a presentar menores proporciones de movilidad ascendente que los nativos que empezaron en dichos niveles. De los migrantes que empezaron como trabajadores manuales no calificados o semicalificados, $69.3 \%$ y $49.2 \%$ respectivamente son móviles ascendentes, frente a $75.2 \%$ y $59.0 \%$ de los nativos. ${ }^{17}$

15 Sobre los aspectos que influyen en las diferencias socieconómicas de migrantes y nativos, consúltense los trabajos de $\mathrm{H}$. Browning y W. Feindt, "Diferencias..." loc cit., 1968; H. Muñoz, O. de Oliveira y C. Stern, "Categorías de...", loc cit., 1971 y; J. Balan, "Migrant-Native Socio-economic Differences in Latin American Cities: A Structural Analysis", en Latin American Research Review, 4:3-29. Una revisión sobre el tema para América Latina puede verse en el trabajo de los autores, "Migraciones internas en América Latina: Exposición y crítica de algunos análisis" en Migración y desarrollo: Consideraciones teóricas, op. cit., 1972. En lo que se refiere a diferencias en la movilidad ocupacional de migrantes y nativos, véase J. Balán y E. Jelín, "Migración a la ciudad y movilidad social: un caso mexicano" en Actas de la Primera Conferencia Regional Latinoamericana de Población, México, El Colegio de México, Vol. I, 1971, pp. 612-616. Sobre migración interna y movilidad en el contexto latinoamericano véase S. Iutaka y W. Bock, "Rural-Urban Migration and Social Mobility: the Controversy on Latinamerica" en Rural Sociology, Vol. 34, Núm. 3, 1969. Además de los anteriores, véase, para México, J. L. Reyna, "Algunas dimensiones de la movilidad ocupacional: Un análisis global", DEMograff́ Y Economía, Vol. 2, Núm. 2, 1968. Asimismo, debe consultarse lo que señala respecto al tema Pablo González Casanova en La democracia en México, México, ERA, 1967, Cap. VI y en "Enajenación y conciencia de clases en México" en Ensayos sobre las clases sociales en México, México, Editorial Nuestro Tiempo, 1968. Por último en el libro de R. D. Hansen, La política del desarrollo mexicano, México, Siglo XXI Editores, 1971, se hacen interpretaciones muy interesantes acerca del significado de la movilidad en el desarrollo del país.

16 Este procedimiento tiene la ventaja de eliminar los efectos de distorsión que se introduce por el nivel al que se hace la incorporación respecto al monto de movilidad. Los que se incorporan en los estratos más bajos tienen una mayor probabilidad estadística de moverse hacia arriba que los que empiezan en los más altos. Por ello, la comparación se hará entre las poblaciones que empezaron en el mismo nivel ocupacional.

17 El dato resulta de interés debido a que en algunos estudios de movilidad ocupacional, los migrantes presentan una mayor proporción de móviles ascendentes por el hecho de que empiezan a trabajar en actividades agrícolas, las cuales, por lo común, son clasificadas en el estrato más bajo. De esta forma, cuando el migrante se traslada a la ciudad y deja de trabajar en este tipo de actividades, automáticamente se registra un ascenso en la escala de estratificación. En este 
b) Los migrantes que comenzaron a trabajar en ocupaciones manuales calificadas o no manuales tienden a presentar mayores proporciones de ascendentes que los nativos que comenzaron en los mismos niveles. Entre los que empezaron en ocupaciones no manuales de bajo nivel $48.5 \%$ de los migrantes son móviles ascendentes, frente a un $29.2 \%$ de los nativos.

\section{Cuadro 3}

Área Metropolitana de la Ciudad de México: Movilidad de la PEA MASCULINA DE 21 A 60 AÑOS DE EDAD SEGÚN CONDICIÓN MIGRATORIA, NÚMERO DE ESTRATOS QUE ASCENDIERON Y ESTRATO DE LA PRIMERA OCUPACIÓN

(Porcientos)

\begin{tabular}{|c|c|c|c|c|c|c|}
\hline & \multicolumn{6}{|c|}{ Estrato primera ooupaoion } \\
\hline & \multicolumn{3}{|c|}{ So manual } & \multicolumn{3}{|c|}{ fianual } \\
\hline & A1to & Medio & Bajo & Califioano & Semicalificado & No oalifioado \\
\hline \multicolumn{7}{|l|}{ Hiprantes } \\
\hline \multirow{2}{*}{$\begin{array}{l}\text { Asoendieron un nivel } \\
\text { Ascondieron dos o más } \\
\text { nivelos } \\
\text { suma }\end{array}$} & 0.0 & 28.4 & 19.3 & 23.1 & 18.0 & 25.2 \\
\hline & $\begin{array}{l}0.0 \\
0.0\end{array}$ & $\begin{array}{r}0.0 \\
28.4 \\
\end{array}$ & $\frac{29.2}{48.5}$ & $\frac{23.5}{46.6}$ & $\begin{array}{l}31.2 \\
49.2\end{array}$ & $\begin{array}{l}44.1 \\
69.3\end{array}$ \\
\hline \multicolumn{7}{|l|}{ Nativos } \\
\hline \multirow{2}{*}{$\begin{array}{l}\text { Asoendieron un nivel } \\
\text { Ascendieron dos o más } \\
\text { niveles } \\
\text { Suma }\end{array}$} & 0.0 & 22.0 & 10.9 & 26.3 & 18.7 & 25.4 \\
\hline & $\begin{array}{l}0.0 \\
0.0\end{array}$ & $\begin{array}{r}0.0 \\
22.0\end{array}$ & $\begin{array}{l}18.3 \\
29.2\end{array}$ & $\begin{array}{l}12.7 \\
39.0\end{array}$ & $\frac{40.5}{59.0}$ & $\frac{49.8}{15.2}$ \\
\hline \multicolumn{7}{|l|}{ Total } \\
\hline \multirow{2}{*}{$\begin{array}{l}\text { Ascendieron un nivel } \\
\text { Ascendieron dos o más } \\
\text { niveles } \\
\text { Suma }\end{array}$} & 0.0 & 24.9 & 13.8 & 25.2 & 18.4 & 25.3 \\
\hline & $\begin{array}{l}0.0 \\
0.0\end{array}$ & $\begin{array}{r}0.0 \\
24.9\end{array}$ & $\begin{array}{l}22.0 \\
35.8\end{array}$ & $\begin{array}{l}16.3 \\
41.5\end{array}$ & $\begin{array}{l}36.5 \\
54.9\end{array}$ & $\begin{array}{l}46.6 \\
71.9\end{array}$ \\
\hline
\end{tabular}

Para apreciar de forma más clara el significado del ascenso se ha desglosado la proporción de éstos en dos partes: la que ascendió un estrato y la que ascendió dos o más estratos.

Si se observa a los que se desplazaron dos o más niveles puede apreciarse que los porcientos varían en la misma dirección de las tendencias señaladas anteriormente:

a) La población migrante que comenzó a trabajar en los estratos más bajos de la estructura ocupacional llega a niveles más altos en una proporción menor que los n̨ativos. Para los trabajadores manuales no calificados se encuentra que $44.1 \%$ de los migrantes, frente a $49.8 \%$ de los nativos, asciende dos o más estratos. Entre los que empezaron como manuales semicalificados las diferencias son más marcadas: $31.2 \%$ de los migrantes y $40.5 \%$ de los nativos asciende dos o más estratos, es decir pasan a ocupaciones no manuales.

trabajo, esta falla metodológica se evita en parte, porque las actividades agrícolas están jerarquizadas a lo largo de todos los estratos. Por ejemplo, en el estrato 6 se encuentran los grandes propietarios agrícolas que tienen más de 11 personas empleadas, mientras que en el estrato 1 se encuentran los peones, jornaleros y asalariados no propietarios, etc. 
b) Al analizar la población que se incorpora en ocupaciones manuales calificadas y no manuales bajas, encontramos que los migrantes recorren dos o más niveles hacia arriba en proporción superior a la de los nativos. Así, se tiene que $23.5 \%$ y $29.2 \%$ de migrantes, frente a $12.7 \%$ y $18.3 \%$ de nativos, respectivamente, ascendieron dos 0 más estratos (véase el cuadro 3 ).

En síntesis, las diferencias entre población migrante y nativa varian según el nivel de la primera ocupación: los migrantes que empezaron su vida activa en los estratos más bajos, como vendedores ambulantes, obreros no calificados o semicalificados, tienden a presentar una movilidad menor, tanto en términos de las proporciones que ascendieron como en términos del número de estratos que recorrieron, en comparación con los nativos que empezaron en los mismos niveles; los migrantes que comenzaron entre los trabajadores manuales calificados y entre los no manuales, como los obreros calificados de la producción, los agentes de ventas y el personal administrativo, presentan como tendencia una mayor proporción de ascendentes y una mayor proporción que recorrió dos o más estratos hacia arriba, en comparación con los nativos del mismo nivel.

\section{ANÁlisis de laS TENDENCIAS DE LA MOVILIDAD OCUPACIONAL POR COHORTES}

Un análisis por cohorte de nacimiento resulta relevante porque es posible que existan diferencias en el nivel de la primera ocupación y en la movilidad de cada cohorte debido, entre otras causas, a las transformaciones generales de la estructura ocupacional en conjunto, asociadas al desarrollo urbano-industrial de la Ciudad de México. Sin embargo, debe apuntarse que por el momento no se pretende estudiar los cambios estructurales en intervalos de tiempo delimitados y su impacto en el comportamiento de cada cohorte, sìno más bien describir este último.

El análisis de los datos sobre la distribución de la primera ocupación de migrantes y nativos, por cohortes de nacimiento (véase el cuadro 4) permite señalar que la población que ingresó más recientemente al mercado de trabajo, se ha incorporado a ocupaciones de niveles más altos en proporciones menores que las cohortes más antiguas. Esta tendencia se aprecia de forma más clara entre la población migrante, ya que para la cohorte de nativos nacida entre 1940 y 1949 existe un aumento en la proporción de personas $(23.7 \%)$ que han ingresado en las ocupaciones no manuales de bajo nivel, comparativamente con las cohortes anteriores de nativos.

Cuando se compara a los migrantes con los nativos, parece que la situación de los primeros tiende a deteriorarse en relación a estos últimos. La diferencia en la proporción de migrantes y nativos que empezaron a trabajar en ocupaciones manuales no calificadas, aumenta en la cohorte más joven $(61.5 \%$ y $46.2 \%$ respectivamente), donde llega a un $15.3 \%$. Así, es posible suponer que los grupos más jóvenes de nativos mejoran sus posibilidades de ingreso a la fuerza de trabajo, en relación con los migrantes. Esto puede deberse a que en los últimos años la proporción de migrantes que proviene de zonas rurales es más elevada y por tanto su origen y su primer trabajo tienden a ser fundamentalmente agrícolas. 


\section{Cuadro 4}

Distribución de la PEA Masculina de 21 a 60 años de edAd SEgúN CONDICIÓN MIGRATORIA, COHORTES DE NACIMIENTO Y ESTRATO DE LA PRIMERA OCUPACIÓN

(Porcientos)

\begin{tabular}{|c|c|c|c|c|c|c|}
\hline & \multicolumn{6}{|c|}{ Estrato primera ooupacion } \\
\hline & \multicolumn{3}{|c|}{ No manual } & \multicolumn{3}{|c|}{ Manual } \\
\hline & Alto & Medio & Bajo & Calificado & Semicalificado & No valificado \\
\hline \multicolumn{7}{|c|}{ Misrantes } \\
\hline $\begin{array}{l}1910-1919 \\
1920-1929 \\
1930-1939 \\
1940-1949\end{array}$ & $\begin{array}{l}2.8 \\
2.9 \\
2.5 \\
1.7\end{array}$ & $\begin{array}{l}2.2 \\
1.1 \\
1.7 \\
2.1\end{array}$ & $\begin{array}{l}15.2 \\
10.8 \\
13.7 \\
12.7\end{array}$ & $\begin{array}{r}12.7 \\
12.4 \\
9.7 \\
9.1\end{array}$ & $\begin{array}{r}8.8 \\
11.4 \\
9.7 \\
12.8\end{array}$ & $\begin{array}{l}58.3 \\
61.3 \\
62.7 \\
61.5 \\
\end{array}$ \\
\hline \multicolumn{7}{|l|}{ Yativos } \\
\hline $\begin{array}{l}1910-1919 \\
1920-1929 \\
1930-1939 \\
1940-1949\end{array}$ & $\begin{array}{l}2.4 \\
1.9 \\
2.8 \\
0.6\end{array}$ & $\begin{array}{l}3.9 \\
0.5 \\
1.5 \\
1.8\end{array}$ & $\begin{array}{l}13.8 \\
16.6 \\
14.3 \\
23.7\end{array}$ & $\begin{array}{l}25.4 \\
16.6 \\
15.7 \\
14.5\end{array}$ & $\begin{array}{l}9.0 \\
10.3 \\
15.2 \\
13.2\end{array}$ & $\begin{array}{l}45.4 \\
54.0 \\
50.6 \\
46.2 \\
\end{array}$ \\
\hline
\end{tabular}

a Las cohortes representan $14.9 \%, 22.5 \%, 29.8 \%$ y $32.7 \%$ de la población migrante, respectivamente.

b Las cohortes representan $8.2 \%, 16.6 \%, 28.1 \%$ y $47.0 \%$ de la población nativa, respectivamente.

Lo anterior, junto con las características del desarrollo del país y de la Ciudad de México en los momentos específicos en que la población se fue incorporando a la fuerza de trabajo, puede explicar las diferencias entre migrantes y nativos.

Si la hipótesis sobre la selectividad socioeconómica decreciente de los flujos migratorios es correcta, esto es, si los migrantes recientes cuentan con bajo nivel educativo y falta de experiencia en trabajos no agrícolas, debido a la mayor contribución que aportan las zonas rurales más atrasadas a la migración, se esperaría que las tendencias diferenciales de movilidad entre migrantes y nativos se acentuaran para las cohortes más jóvenes. En otras palabras, sería de esperar que las cohortes más jóvenes de migrantes hayan experimentado una menor movilidad con relación a las cohortes respectivas de nativos.

Si se considera únicamente aquellos casos en que los migrantes presentan menor movilidad que los nativos (véanse las cifras subrayadas del cuadro 5) podemos afirmar que:

a) La movilidad de los migrantes es menor para la cohorte que nació entre 1910 y 1919, que la de los nativos en los casos en que iniciaron su vida activa en posiciones no manuales medias y manuales semicalificadas. Si se observa el cuadro 4 se podrá apreciar que la proporción de migrantes que tuvieron una menor movilidad que los nativos representa un $11 \%(2.2 \%+8.8 \%)$ de la población migrante de esta cohorte.

b) Para la cohorte que nació entre 1920 y 1929 se advierte que en ningún nivel los migrantes tuvieron una menor movilidad que los nativos.

c) Para la cohorte que nació entre 1930 y 1939, los migrantes tienen una menor proporción de móviles ascendentes en comparación con los nativos para aquellos que comenzaron a trabajar en los niveles ma- 
Cuadro 5

Distribución porcentual de las personas móviles de la PEA masculina DE 21 A 60 AÑOS DE EDAD, SEGÚN CONDICIÓN MIGRATORIA, ESTRATO DE LA PRIMERA OCUPACIÓN Y COHORTES DE NACIMIENTO

\begin{tabular}{|c|c|c|c|c|c|c|}
\hline & \multicolumn{6}{|c|}{ Estrato primera ooupeoión } \\
\hline & \multicolumn{3}{|c|}{ No manual } & \multicolumn{2}{|r|}{ Manual } & . \\
\hline & Al to & Modio & Bajo & Califícado & Somioalificado & No ollifioado \\
\hline \multicolumn{7}{|l|}{ XIgranteog } \\
\hline $\begin{array}{l}1910-1919 \\
1920-1929 \\
1930-1939 \\
1940-1949\end{array}$ & $\begin{array}{l}0.0 \\
0.0 \\
0.0 \\
0.0\end{array}$ & $\begin{array}{l}\frac{14.3}{0.0} \\
\frac{16.0}{54.4}\end{array}$ & $\begin{array}{l}66.1 \\
45.6 \\
47.5 \\
41.8\end{array}$ & $\begin{array}{l}69.0 \\
44.1 \\
49.3 \\
32.1\end{array}$ & $\begin{array}{l}\frac{75.6}{59.8} \\
37.6 \\
10.0\end{array}$ & $\begin{array}{l}70.6 \\
77.1 \\
66.8 \\
62.7 \\
\end{array}$ \\
\hline \multicolumn{7}{|l|}{ Intivon } \\
\hline $\begin{array}{l}1910-1919 \\
1920-1929 \\
1930-1939 \\
1940-1949\end{array}$ & $\begin{array}{l}0.0 \\
0.0 \\
0.0 \\
0.0\end{array}$ & $\begin{array}{r}26.8 \\
0.0 \\
66.4 \\
0.0\end{array}$ & $\begin{array}{l}50.8 \\
28.4 \\
44.6 \\
21.7\end{array}$ & $\begin{array}{l}59.2 \\
38.2 \\
43.0 \\
30.4\end{array}$ & $\begin{array}{l}99.3 \\
47.3 \\
61.9 \\
55.7\end{array}$ & $\begin{array}{l}59.4 \\
75.6 \\
81.5 \\
73.8\end{array}$ \\
\hline
\end{tabular}

nuales semi y no calificados y entre los no manuales de nivel medio. La proporción de migrantes que tuvieron una menor movilidad que los nativos representa un $74.1 \%(1.7 \%+9.7 \%+62.7 \%)$ de la población migrante de esta cohorte.

d) Para la cohorte que nació entre 1940 y 1949, la proporción de móviles migrantes es menor que la de nativos, para aquellos cuya primera ocupación fue manual semi y no calificada. Estos migrantes representan un $74.3 \%(12.8 \%+61.5 \%)$ del total de la cohorte de migrantes según los datos del cuadro 4.

Los resultados de la movilidad por estrato de incorporación para diversas cohortes de nacimiento sugieren que las posibilidades de ascenso para los migrantes eran tan buenas o mejores que las de los nativos, para los nacidos entre 1910 y 1929. Asimismo, se sugiere que probablemente exista un cambio en dichas oportunidades al nivel de las posiciones más bajas, ya que en las dos cohortes más jóvenes, los migrantes cuya primera ocupación fue entre los manuales semi y no calificados, presentan una menor proporción de ascendentes que los nativos, lo que parece estar asociado con características diferenciales de las cohortes migratorias.

Así, los datos permitirían concluir, en forma preliminar, que los migrantes que inician su vida activa en épocas recientes, en los sectores manuales calificados y no manuales - lo cual puede estar relacionado a un cierto nivel educativo- son los que tienen más posibilidad de mejorar su posición en la estructura ocupacional en la Ciudad de México.

Por último, deben señalarse otras tendencias que se derivan de los datos del cuadro 5. Por un lado, entre la población migrante, a excepción de los que empezaron a trabajar en el nivel no manual medio, se observa que la proporción de móviles ascendentes tiende a disminuir en el tiempo, a medida que la cohorte de nacimiento es más joven y por otro lado, cuando se considera a los nativos, se advierte que la cohorte nacida entre 1930 y 1939 tuvo más movilidad que las cohortes nacidas entre 1920-1929 y'1940-1949.

Ambos resultados sugieren que la cohorte más joven contó con menores posibilidades de movilidad ascendente, lo que puede estar asocia- 
do tanto a una mayor rigidez de la estructura ocupacional como a características diferenciales de cada cohorte.

Para confirmar una hipótesis como la anterior sería necesario llevar a cabo un análisis de los cambios ocurridos en la estructura ocupacional, en la creación de empleos por ramas y sectores de actividad y en las exigencias requeridas para ocupar una posición dada, junto con un análisis de las características demográficas y socioeconómicas —edad de entrada a la fuerza de trabajo, nivel de instrucción- de cada cohorte de migrantes y nativos. Sólo a través de este tipo de análisis se puede aclarar y explicar el comportamiento diferencial de cada cohorte.

\section{COMENTARIos, Finales}

Debido a que la mayor o menor proporción de movilidad observada puede depender en gran parte de aspectos metodológicos ${ }^{18}$ ligados a la medición del fenómeno, no se ha puesto énfasis en el análisis cuantitativo de la movilidad y en sus tasas globales, sino en las diferencias entre migrantes y nativos. En este sentido, podría decirse que si bien los métodos de análisis pueden influir en las proporciones totales de la población móvil, no deben modificar las tendencias básicas que se han observado respecto a las diferencias entre estas dos poblaciones.

Los resultados permiten señalar que la movilidad ascendente se presenta a lo largo de toda la estructura ocupacional para las diferentes cohortes. Los migrantes en cada uno de los niveles ocupacionales se ven sujetos a movilidad ascendente aunque ésta sea menor que la de los nativos en las cohortes nacidas de 1930 a 1949, principalmente para los que se incorporaron a la fuerza de trabajo en posiciones manuales no calificadas y semicalificadas.

Según las tendencias que presentan los datos, se puede afirmar que hasta el momento la movilidad hacia estratos superiores ha sido un fenómeno presente en el proceso de desarrollo de la Ciudad de México. Sin embargo, como se ha visto, este fenómeno coexiste con el de la marginalidad de un sector de la población. Ello puede deberse a que el proceso de creación de empleos no ha sido lo suficientemente dinámico para absorber a mayores cantidades de migrantes y nativos a niveles no marginales. ${ }^{19}$

Asimismo, se puede señalar que parte de la población que comenzó su vida activa en ocupaciones de los estratos más bajos, en los sectores

18 Así, por ejemplo, la alta proporción de móviles ascendentes puede estar afectada por la forma como fue medida la movilidad, ya que al comparar la primera ocupación con la actual se toma en cuenta el mayor lapso de tiempo transcurrido en la vida activa $\mathrm{y}$, por tanto, son más elevadas las posibilidades de alcanzar niveles ocupacionales de más alta jerarquía. Asimismo, debido a que las primeras ocupaciones pertenecen por lo regular a estratos de bajo rango, la proporción de móviles ascendentes tiende a ser mayor. De igual modo, debe insistirse en que la proporción de móviles ascendentes puede depender del número de categorías o estratos que se utilizan en el análisis, ya que entre más categorías se tengan mayor es también la movilidad que resulta.

19 En un trabajo sobre la Ciudad de México se indica que entre 1960 y 1970 la creación de empleos para ambos sexos experimentó una reducción con respecto al decenio anterior. Véase Enrique Contreras, "Migración interna y oportunidades de empleo en la Ciudad de México", op. cit., 1972. 
marginales, pudo superar esta situación, con tendencia más marcada entre los nativos que entre los migrantes, sobre todo para las cohortes nacidas de 1920 a 1949. Para la cohorte de 1910 a 1919 la tendencia es opuesta.

En este sentido, la marginalidad ocupacional puede ser un hecho transitorio para una parte de la población, debido a las oportunidades de movilidad ascendente. Sin embargo, la marginalidad como proceso estructural depende de las tendencias que asume en su conjunto el proceso de desarrollo a nivel nacional así como de la mayor o menor tasa de creación de empleos y del volumen de mano de obra disponible en la ciudad. Información adicional acerca de estos elementos permitiría establecer con mayor precisión las relaciones entre la migración, la movilidad y la marginalidad.

De esta manera, se necesita estudiar los movimientos interregionales e intersectoriales de población con el fin de observar los procesos de formación y los cambios de la fuerza de trabajo en la ciudad.

La migración interna y la movilidad ocupacional pueden ser analizados como parte de un solo proceso de cambio estructural que hace referencia a los movimientos intra e intersectoriales de la mano de obra dentro del marco de desarrollo global de la sociedad. Lo anterior sugiere que el estudio de las diferencias entre migrantes y nativos en los puntos de destino no debe constituir el centro del análisis, aunque se reconoce que puede ilustrar los cambios estructurales sobre todo cuando se basa en la comparación de diferentes cohortes poblacionales.

\section{APENDICE METODOLOGICO *}

Para llevar a cabo el estudio de la movilidad se formaron estratos ocupacionales de acuerdo a los procedimientos que se indican a continuación.

El estrato ocupacional se refiere a la posición relativa que tienen los individuos en la estructura ocupacional. El conjunto de los estratos constituye la jerarquía de las ocupaciones de los entrevistados. La construcción de los estratos se hace necesaria para resumir todas las ocupaciones en un número más pequeño de categorías y para medir la movilidad. La ordenación de las ocupaciones en una escala permite conocer qué individuos logran ascender o mejorar su posición, cuáles descienden y cuáles permanecen en el mismo nivel, en este caso, desde el momento en que empezaron a trabajar hasta la fecha en que se hizo la encuesta.

Los criterios empleados para jerarquizar las ocupaciones de la población son los siguientes: a partir de la ocupación de las personas se formó un conjunto de grupos ocupacionales dentro de los cuales se diferenciaron las primeras por: propiedad o utilización de ciertos bienes en el trabajo, posición en la ocupación, número de personas remuneradas que se empleañ o el número de personas que se tienen bajo las órdenes directas e indirectas. Para el caso particular de los trabajadores manuales, además de los ya mencionados se aplicaron otros de orden general derivados del carácter de la ocupación: a) la definición de sus actividades, b) el grado de responsabili-

* La metodología que se presenta fue elaborada para uso general en los análisis de la encuesta de Migración interna, estructura ocupacional y movilidad social en el área metropolitana de la Ciudad de México por los autores conjuntamente con Claudio Stern. 
dad por el uso o manejo de maquinaria y, c) requisitos para desempeñar el trabajo en términos de las exigencias o capacitación para realizar las tareas.

Los estratos se utilizaron para clasificar la primera ocupación de las personas y la que actualmente desempeñan. La movilidad se registró cuando hubo un cambio de estrato. Por ejemplo, se dice que la movilidad es ascendente cuando dicho cambio significó pasar de un estrato inferior a otro superior.

Con fines exclusivamente de presentación se ha denominado a los estratos 6,5 y 4 como no manuales y a los estratos 3, 2 y 1 como manuales ya que incluyen básicamente este tipo de ocupaciones.

A continuación se presenta la lista de estratos y algunos ejemplos de las ocupaciones que contienen, así como las proporciones correspondientes de la PEA en cada nivel.

Estrato 6: Legisladores y funcionarios públicos de alto nivel, personal directivo de empresas con más de 11 personas a su cargo, funcionarios públicos con más de 11 personas a su cargo, técnicos y subprofesionales que tienen más de 10 personas a su cargo o que son independientes y utilizan local y emplean personal, propietarios de empresas no agrícolas que emplean más de seis personas y propietarios de empresas agrícolas que emplean más de 11 personas, etc.

Estrato 5: Técnicos, subprofesionales, funcionarios públicos y personal directivo que tienen de 1 a 9 personas a su cargo, agentes, vendedores y trabajadores administrativos que tienen más de 5 personas a sus órdenes, técnicos y subprofesionales independientes (resto), propietarios de empresas no agrícolas que emplean de 6 a 10 personas, etc.

Estrato 4: Técnicos y subprofesionales dependientes (resto), personal directivo, funcionarios públicos y trabajadores administrativos que no tienen personas a su cargo, propietarios de empresas no agrícolas que no emplean personal, supervisores de los servicios, la construcción y de la producción que tienen 5 o más personas a su cargo, obreros y artesanos de la producción, propietarios que emplean de 1 a 5 personas, propietarios agrícolas que emplean de 1 a 5 personas, etc.

Estrato 3: Obreros calificados de la producción y de la construcción dependientes, obreros calificados de la producción y de la construcción propietarios que no emplean personal, operadores de vehículos de motor, etc.

Estrato 2: Obreros semicalificados de la producción y de la construcción, conserjes y mozos de oficinas, agricultores y ganaderos no propietarios con 1 a 5 personas a su cargo, etc.

Estrato 1: Trabajadores no calificados de los servicios, obreros no calificados de la producción y de la construcción, trabajadores del campo, dependientes, sin personal o independientes no propietarios, vendedores ambulantes, etc.

La distribución de la PEÁ masculina de 21 a 60 años de edad por estrato de la ocupación actual es: no manuales altos $(8.6 \%)$, medios $(7.1 \%)$, bajos $(24.0 \%)$; manuales calificados $(21.3 \%)$, semicalificados $(19.9 \%)$, no calificados $(19.1 \%)$. 